\title{
Implementation of a computer algorithm for an interactive 3D CAD generation of tensegrity structures
}

\author{
Jason E. Charalambides and Katherine A. Liapi
}

\begin{abstract}
Tensegrity is a technology that can be applied to structures and its use can influence the construction time efficiency and project management in general. A significant drawback for a systematic application of tensegrity structures in the construction industry is the particularly complex geometry that engineers and architects have to generate in a two or three dimensional virtual or physical environment. This paper discusses a computer based utility that will facilitate the design professional to devise and construct a specific morphological variation of tensegrity structure systems. This utility applies to a recently developed building technology application which allows methods of forming a structure based on the rapid assembly of existing tensegrity units, permitting variability in the overall structure's form and dimensions. The development of the proposed utility was based on a methodology that identified and included parameters that can be associated to the schematic design and design development phases of a design project. Various methods of processing data, each of which may correspond to the needs of the designer, are enabled within the interface; domical, vaulted and slab configurations are the principal geometries addressed. Emphasis in the software application was placed on the development of a user friendly interactive environment that assists the generation and modification of the numerical data, and allows unobtrusive regenerations of alternate solutions. The developed utility - an automation tool - is to be used for decision making, enabling the user to iterate processes and make fine adjustments that will satisfy the agenda of parameters set by the project designers. The software application has been validated with a series of tests, briefly presented in the paper.
\end{abstract}

\section{TENSEGRITY STRUCTURES FOR BUILDING DESIGN}

Tensegrity structures for application in building design can be of various geometric forms and morphological types.

Jason. E. Charalambides is Assistant Professor at the Frederick Institute of Technology, Nicosia, Cyprus (email:j.charalambides@alum.calberkeley.org, phone: ++ 357- 22377946)

Katherine A. Liapi is Assistant Professor of Architectural Engineering at the University of Patras, Greece, and Adjunct Assistant Professor of Civil Engineering at the University of Texas at Austin (e-mail: kliapi@mail.utexas.edu)
Yet it can be maintained that Tensegrity structures in general are based on the principle of disjoint connections. This is characteristic of a structural system in which rigid members, such as bars, are not connected directly to each other, but to cables that usually form a continuous network. Although a few researchers and designers disregard this condition, based on the definitions given by Fuller [1] who first came up with the term, as well as of Pugh [2], Emmerich [3], Hanaor [4], and others who contributed to the research in the field, discontinuity of compressive components remains central to the tensegrity concept.

\section{A. Basic Tensegrity Morphologies}

Tensegrity structures can be arranged in a single and a double layer fashion. Single layer morphological types were extensively studied by Oren Vilnay [5]. The single layer geometry, lacking the depth of its double layer counterpart, is characterized by typical "membrane" self supported structure issues of stability and vibrations, a subject Vilnay specifically engages on in his work on analysis and design applications. Double layer tensegrity structures occur from the assembly of tensegrity units of simple geometry. Based on this, double layer tensegrity structures are modular in conception and can therefore support pre-fabrication and pre-assembly. Research on double layer tensegrity forms was carried on extensively by Rene Motro who employed tensegrity units of square base [6]. Motro, in order to simplify the geometry of tensegrity structures, connected tensegrity units at nodal points; this resulted in structures that only partially fulfill the condition for disjointed connections between members.

Ariel Hanaor engaged more on the triangular based formation, that is, on structures that occur from the disjointed assembly of tensegrity units of triangular base, and gave emphasis on the development of the geometry of tensegrity structures of spherical shape. Hanaor also conducted load tests and structural analysis studies [7]. A morphology similar to Hanaor's was used by Allain Chassagnoux of the Ecole d'Architecture de Nantes in France. Chassagnoux developed a computer program that calculates displacements on tensegrity structures using the finite elements method [8].

On the morphological type of square base double layer tensegrity structures, research has been carried on by 
Katherine Liapi at the University of Texas at Austin, Department of Civil Engineering [9]. Liapi proposes curved configurations from identical prismatic tensegrity units of square base instead of the triangular base units that Hanaor and Chassagnoux consider.

The objective set by Liapi was the possibility of generation of variable curvatures in two perpendicular axes vis-à-vis the possibility of only one type of curvature that the triangular based units can produce as shown in the example of the works of Hanaor and Chassagnoux in Fig. 1. Departing from the square base morphology, Liapi also developed a building technology application which makes possible the rapid assembly of tensegrity structures from pre-fabricated and preassembled tensegrity modules. This method permits reusability of existing modules while allowing for variability in the overall structure's form and dimensions [10].

The study presented in this paper addresses the double layer tensegrity structures composed of disjointed tensegrity units of square base. Those can be used in conjunction with the recently developed technology that allows the generation of structures of various configurations. One of the advantages of this new technology is also the option of reusing existing units from previously disbanded structures.

\section{B. Square base unit structure: Geometric resolution and basic configurations}

Tensegrity structures are characterized by particularly complex geometry that engineers and architects have to generate in a two or three dimensional virtual or physical environment. This is a drawback for a systematic application of tensegrity structures in the construction industry. Although patterns and general rules for the assembly of double layer tensegrity networks already exist, the geometry that occurs when these patterns are applied is very hard to determine and to graphically generate.
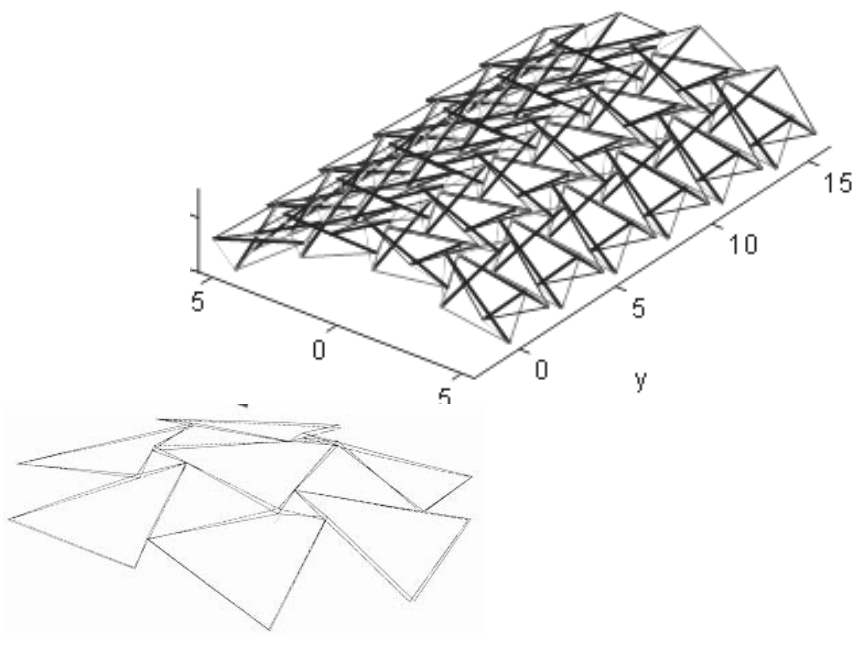

Fig. 1. A Vault form designed using double layer square based tensegrity units vs a spherical arrangement of the upper bases of triangular base tensegrity units.
Fundamental geometric principles and conditions that determine tensegrity unit adjacency that apply to tensegrity structures of either spherical or cylindrical shapes have been identified and demonstrated by Liapi [9] (Fig. 2a). The application of these principles and conditions have made possible the generation of the geometry and the visualization of tensegrity structures of domical (spherical) shape [11]. For the generation of the geometry of tensegrity structures of cylindrical shape, the development of a variation of the basic unit adjacency principle and conditions was required (Fig. $2 b)$. These new adjacency conditions have made possible the investigation of the spatial configuration of tensegrity structures of vaulted (cylindrical) form [12] .

However the developed methods for the generation of the dome and vault geometry remain a time consuming process when form exploration requires the generation of a large number of CAD models that will fulfill various design scenarios and constraints. Taking into account the parametric character of these structures, as well as the difficulty in generating several configurations by directly applying geometric rules and processes, it becomes obvious that there is need for a tool that would come to the aid of a designer by automatically generating 3D CAD models of tensegrity structures. It should be noted that even a seemingly minor alteration of the overlapping section of the cables of adjacent units can cause substantial changes to the form of a structure. Thus, a computer utility which will automatically generate tensegrity formations is proposed.
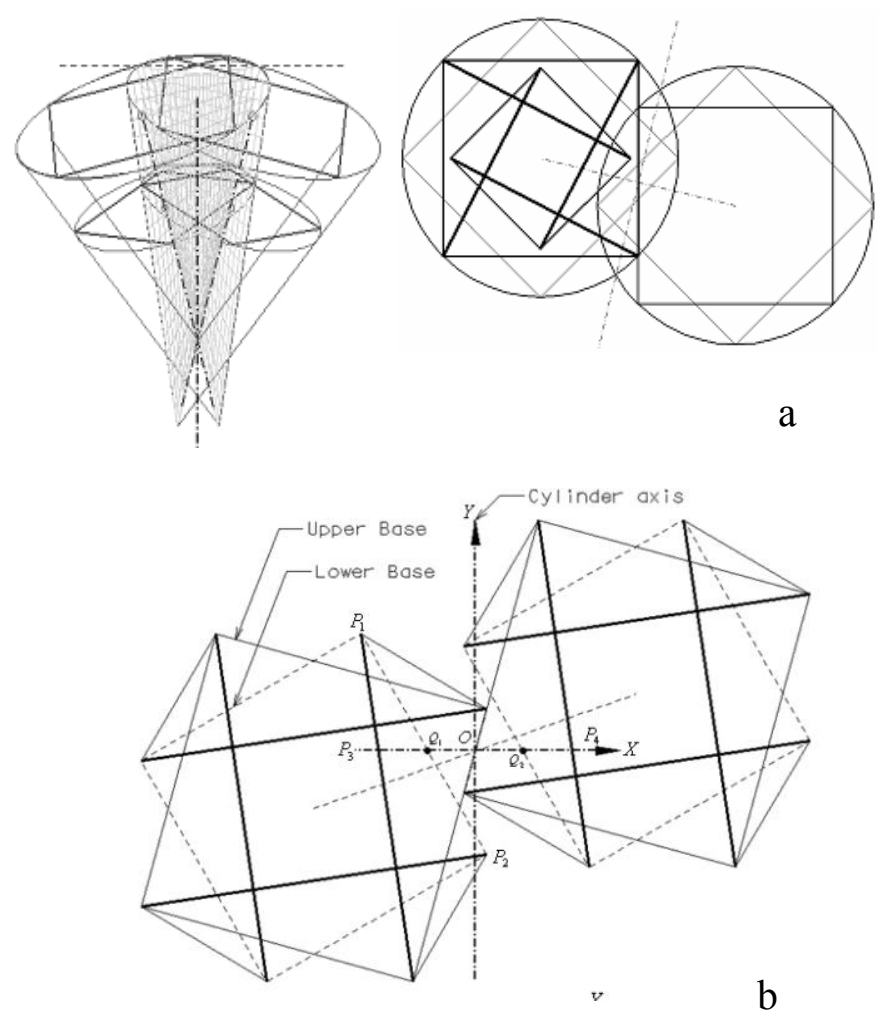

Fig. 2. Tensegrity unit adjacency conditions for: a) domical form and $b$ ) vaulted form 


\section{AN INTERACTIVE AUTOMATED FORM GENERATION APPROACH}

The main objective of the developed research is the generation of a tool that will assume all the calculative processes for the generation of tensegrity structures in CAD form, in order to allow the designer to engage only into the decision making processes. This objective could be fulfilled through the development of a computer program characterized by its interactive method of operation and its integrability to available CAD packages. The main features of the proposed utility are described below.

\section{A. Supported geometries}

The above mentioned morphological type, arranged in a modular fashion, allows single and double curvature structural forms to be generated. The arrangement of the units can be set on Cartesian or spherical coordinates. The double layer tensegrity structures based on the square unit allow the generation of three types of configurations: a) dome, b) vault, and c) flat slab as seen in Fig. 3. Another potential formation of a variable curvature along the $\mathrm{x}$ and $\mathrm{y}$ axes as seen in Fig. 4 is under investigation.

The formation and all the variations of these forms are based on the method of unit connection. The developed expressions for the vault and slab configurations can always generate a final geometry with no overlapping units or any other discrepancies. The geometric relations of the dome configuration however allow the generation of overlapping units toward the outer edges as seen in Fig. 5, when the curvature is large.

In an effort to transfer the geometric relations into a series of equations, the geometric principles that apply to the method of unit connection were applied, while additional relations had to be developed. By applying the geometric principles discussed earlier [11], the diagrams shown in Fig. 6 occur.

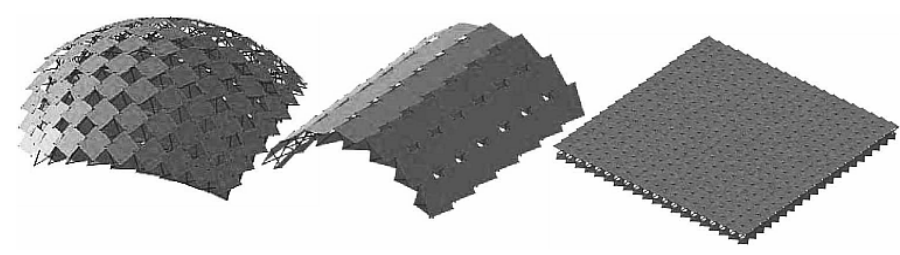

Fig. 3. Three basic forms generated with the use of double layer square base tensegrity units - dome, vault and slab.

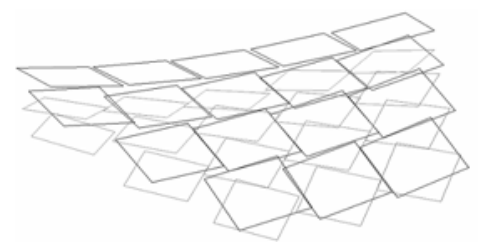

Fig. 4. A double curvature formation under investigation.
In the diagram on the right of Fig. 6 the line that connects the centers of the top squares is the projected axis along which the rotation between every two elements takes place. In order to generate a dome form, the centers of the upper and lower squares should form an axis - indicated as RAX (Rotation axis) - that leads to the center of a spherical form. For the vault formation, the rotation of the RAX, as indicated in Fig. 7 , is offset and a series equation is applied to calculate the span of the structure. With this offsetting method no overlapping of surfaces occurs among the structure units. The relation applied to the vault can mathematically be applied to the slab configuration as well, even though the latter is designed to be preset for no rotation. This can explain why the discrepancy in the dome form takes place as a set of squares is rotated along a spherical set of coordinates.

This discrepancy takes place at all levels because a sphere can not be formed by squares. Yet, the effect is less evident in smaller curvatures. As expected, such configuration can cause problems in the generation of an actual structure, or even a designed structure may be impossible to construct. No special warnings by the developed algorithm were judged necessary. The generated result is immediately visible to the designer within the environment of the computer, and therefore there was no need to set special warnings. For the generation of the vault form, the basic adjacency conditions as defined by Liapi and Kim [12], have been applied and additional relations have been developed as shown in Fig. 7. These relations were set in a series of equations that integrate all interrelated parameters that control this type of formation.

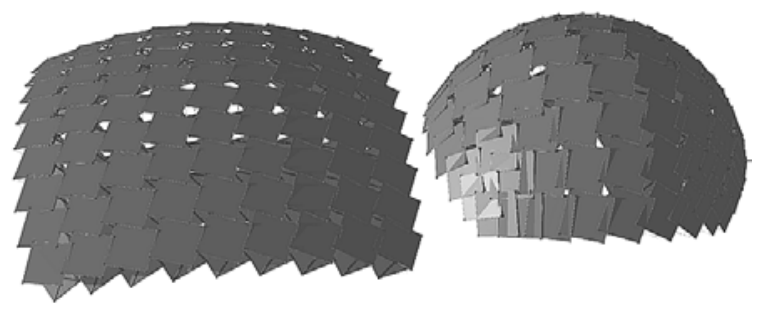

Fig. 5. Two dome models set at rotations of $48^{\circ}$ and $135^{\circ}$. Severe unit overlap is noted on the second model.

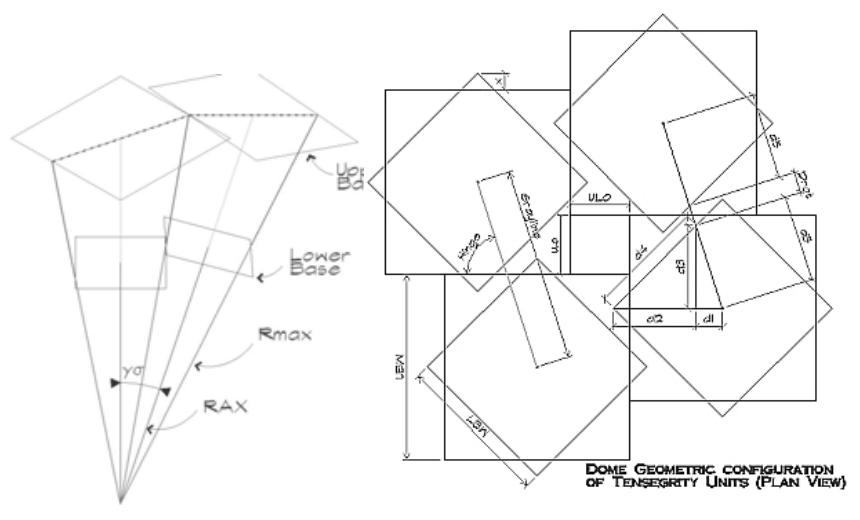

Fig. 6. Plan of a four unit cluster set for dome formation before rotation, and diagram of two units in rotation 

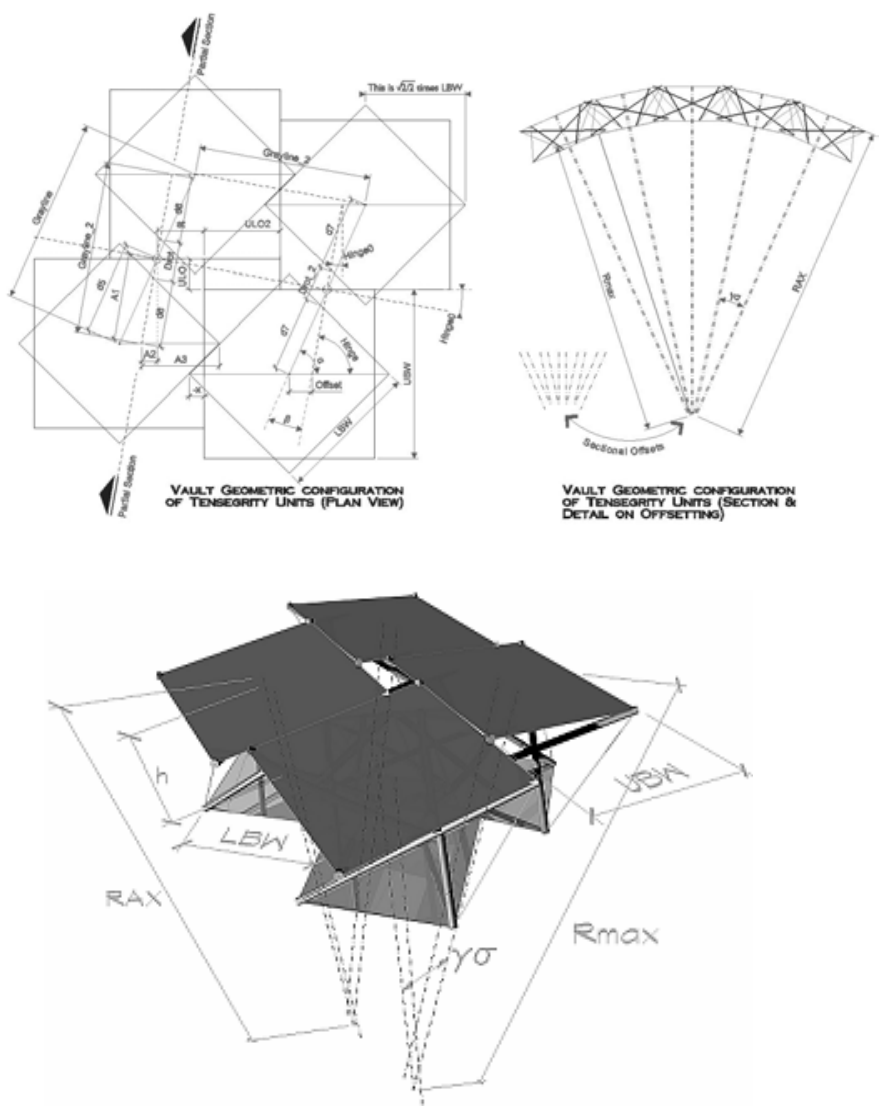

Fig. 7. Diagrams of four unit cluster in vault configuration and Section of a vault structure after rotation.

\section{Design processes and Options}

The mathematical solution of the geometry and connections already established an analytical method to follow toward the construction of a virtual model. Specifically each element's characteristics were established in a linear fashion, and the relations follow a seamless process.

In addition various methods of processing data, each of which may correspond to the needs of the designer should be enabled within the interface, have been developed. Taking into account that designers need to employ various methods for the final product they envision, the association of the proposed utility to the schematic design and design development phases of a design project would be particularly advantageous. Thus the proposed utility should not be limited to only one method of solving the geometric relations but rather address a number of possible scenarios that can arrange a different set of priorities and objectives.

Specifically, three basic processes that can be followed in the design of tensegrity structures have been developed. The first process is based on the initial analysis of the elements and the way they are connected. Thus the process starts from the unit geometry and the connections among the units. Those data determine the rotation among the units, and since the number of units is defined, the dimensions of the structure are calculated. Nevertheless, in terms of numerical relations, the results of the first method can not be predictable. That could cause some difficulty to designers whilst processing unit geometry to generate the general structure dimensions. The results of the process, being available to be reused, can be adjusted as new input. Therefore, the designer can readjust dimensions and the "opening ratios" of the units in order to regulate the dimensions of the entire structure. This process is the preferred one when a designer already has available prefabricated elements that can be used for a structure. It is also very useful in making minor or fine adjustments to the results that another process already yielded.

The second process, unlike the first one, allows the designer to determine the dimensions of the structure and the program computes the dimensions of the units. The points of connection between the units, however, also need to be determined by the designer in which case some guess values will need to be applied. This process is more useful at a Design Development stage of a project where prefabricated preassembled elements are not available and can be designed and set within the specifications of the project.

The first and second processes were applied to all three geometric configurations. The third process was not applicable for the slab design due to its simpler geometry. It combines parts of both previous processes but it is the only one where the designer has to determine neither the connection points or opening ratios, nor the number of units. The basic unit geometry and the overall structure geometry are determined by the user. The process will calculate the number of units and the type of connections that will generate the form. The second and third processes, where the structure span was a set objective, generated a serious obstacle to the vault configuration due to the application of series equations. Inversion of a series equation would be unrealistic. A loop sub-process that was applied was named "random progressive" because of the use of the random "rnd" factor by which the error was multiplied in each iteration which would progressively lead to the objective results. The reason the random factor was used was that a non varying set factor could - and during most of the experiments would - lead to an infinite loop where the progressive order would be trapped and the error would alternately once become slightly larger and then become slightly smaller. The random factor however, would be different on every loop, not allowing an exact repetitive function but still adjust the results at a different pace on every iteration.

A fourth process within which an optimization of the configuration for better structural stability was considered but not developed yet. In the generation of the computer program, however, an inactive option for an optimal structural stability method is set. The option will be activated once this part of the algorithm is developed. Furthermore, the arrangement of the different processes is not set according to the timing of their development but according to how important they may be to a designer. Thus, the fourth option that is not developed yet is actually set on the first method tag as seen in Fig. 8. 


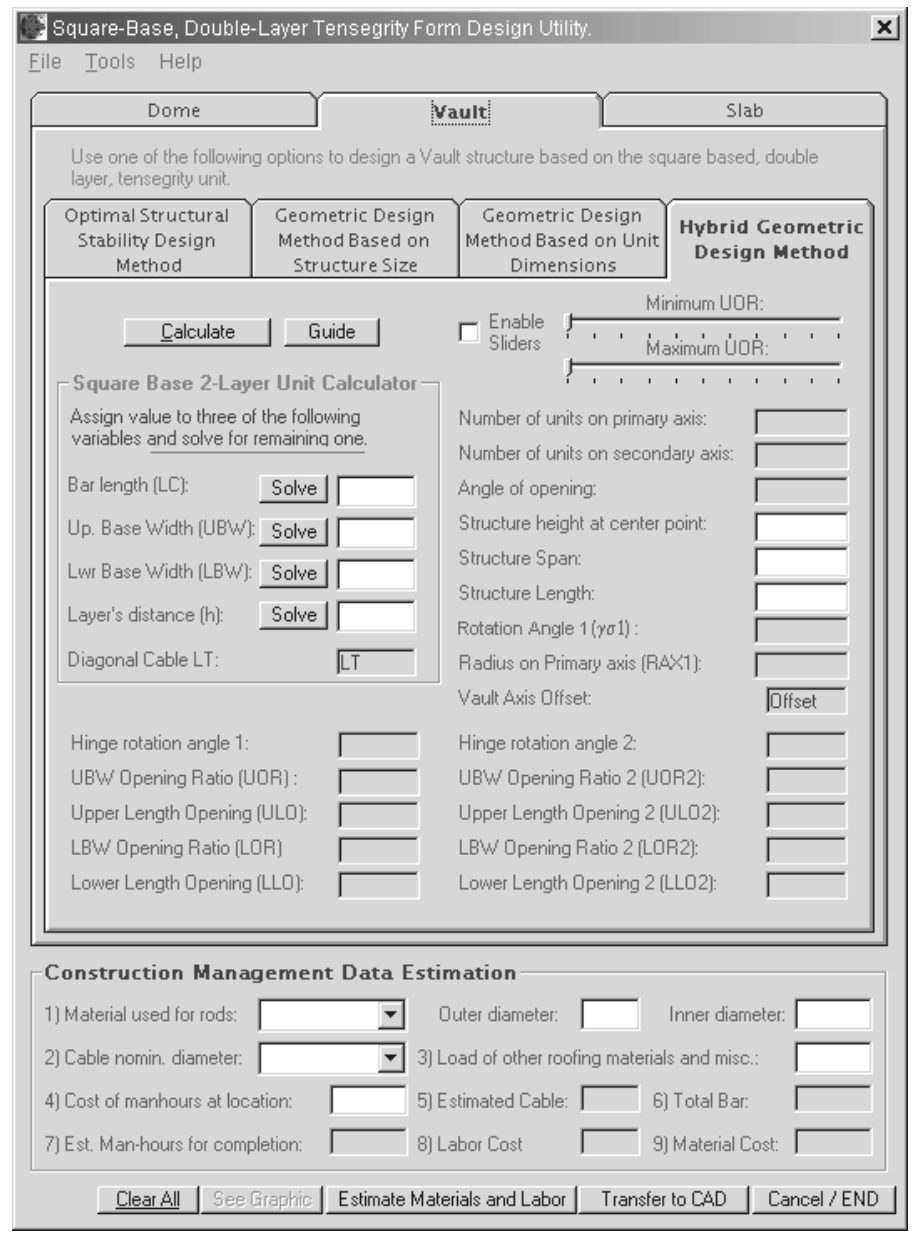

Fig. 8. View of the program's interface on hybrid process for vault structural form generation

\section{ALGORITHM DESIGN PROCESS AND DEVELOPMENT:}

The significant factor in the choice of a computer language was the interactive character of the interface. The user would have to have a set of conditions that can remain available and have the opportunity to alter specific data and determine which data should be recalculated. For these reasons, as well as for the ease of use, the language chosen was Visual Basic. VBA would have been ideal to use as it is available in most current CAD applications, eliminating the issue of communication between two programs. However, VBA's capacity being more limited, lead to the generation of a main application that would perform the primary calculations, and a VBA applet that would operate within the CAD package and that would communicate with the main application. The DLL option was contemplated but rejected because it was considered inefficient. Besides the advantage of a more integrated method of interaction, a DLL option would be more complicated to combine with all commercially available software. The method of data transferred was set through text based files stored in a temporary folder; a much simpler, efficient and reliable method with fewer considerations.

This communication between the main application and a VBA applet could be developed for many available CAD software packages. One chosen for its powerful three dimensional modeling and rendering capabilities was Bentley MicroStation. Within its environment the applet is activated. Through the applet, the main application is launched and a set of data can be transferred to the main application for processing. Once the general geometry is resolved, data related to materials and costs can be calculated and values returned to the user. The absence of a DLL connection imposes that the user applies a command for data export to a temporary folder, and subsequent import of those data to the VBA applet. Once the geometry data is imported, the user can activate the macro command for the generation of the virtual model within the CAD environment.

The raw data of the temporary file generated by the main application determine the number of tensegrity units to be used for the structure, the geometry of the units, angles of connection and connection points. The VBA applet translates all the above mentioned data to macro commands that perform all the modeling routines within the CAD environment. The limitation in the number of command lines that can be used within the VBA can cause some problems when a large number of tensegrity units is needed to cover the span of a structural form. At this point, the command is structured in a way that would produce up to nine units across any curved surface. Non curving surfaces in the slab or vaulted structural forms impose no limit to the number of units that can be used. Thus, the length of a vaulted structure has no ceiling value. The problem of the limitations of the VBA language could be addressed by the development of public command routines. It was not addressed however because it is assumed that up to nine units across the curvilinear direction of any of theses structures should most times be adequate. In a rare case when more series of units would be needed, manual addition of a series by a user can be an option.

\section{TESTING:}

Testing took place to verify the precision of the produced results as well as the stability and effectiveness of the compiled algorithm. The objective is to confirm that even if the user mistakenly enters data that would generate a non buildable structure, the program will not fail, but will either give a warning message to the user or, if the virtual model can be produced, to proceed and allow the user to assess if the structure is buildable or not. The method followed for the testing was set to primarily input data that will generate extreme curvatures, and secondarily, input data at random.

In testing for the generation of all the structural forms, the calculations and the CAD model would be generated within less than a minute including ray-trace antialias rendering. Whenever the number of units was determined by the user, the maximum of nine units across the width of the structure, which is the maximum allowed by the algorithm, was used. In some further testing for comparative purposes, a lesser number of units was produced, but the difference in timing for the generation of the model was within a second. The only 
case where a test fell short was when the hybrid method was applied and the calculated number of units across the structure was eleven. The program produced a structure up to the ninth series because the program was set to operate to that maximum number of series of units. A result like this was anticipated to happen at some stage, and a safety precaution was set within the program to not stall the program but to produce the structure, so that manual addition of sets of extra series could be an option for the user.

\section{CONCLUSIONS}

The goal of the research was to produce a tool that would assume all the calculative processes for the generation of tensegrity structures in CAD form, in order to allow the designer to engage into the decision making processes. The objective was to be fulfilled through the development of a computer program that would be characterized by its interactive method of operation and its integrability to available CAD packages. A computer based utility to automatically generate the geometry of square base double layer tensegrity structures has been developed. The interactive interface allows the user to choose among three processes offered and three geometric forms.

The prepared algorithm that was compiled into a main computer application and an auxiliary applet, provide the user with numerical values that describe the geometry of square base double layer tensegrity structures and allow flexibility of iterative processes and alterations. Beyond the initially set objective, the applications provide the possibility for the iterative processes to take place at multiple levels by providing the user with different methods of data processing to be followed, allowing the user to modify data at will.

An automated generation of virtual models has been achieved through the splitting of the program into a main application and an applet within a CAD environment, which communicate and exchange data. The main application can work as a standalone program that provides numerical results, but in a synergetic manner with the applet, the two can provide a more integrated result of $3 \mathrm{D}$ virtual models. The generated 3D models address only the initial geometry of such structures. The applications have been tested and verified to function seamlessly.

\section{REFERENCES}

[1] Fuller, R. B., Synergetics: Explorations in the geometry of thinking, Macmillan, New York, 1975.

[2] Pugh, A., (1976). An Introduction to Tensegrity, Berkeley, CA: University of California Press.

[3] Emmerich, D.G., "Emmerich on Self-Tensioning Structures", International Journal of Space Structures, 1996, 11(1) \& (2), 29-36.

[4] Hanaor, A.,"Tensegrity Theory and Application", Gabriel, J. F. ed., Beyond the Cube, 385-408, John Wiley \& Sons, Inc., New York, 1998.

[5] Vilnay, O., (1990). Cable Nets and Tensegric Shells: Analysis and Design Applications, NY: Ellis Horwood.

[6] Motro R., "Tensegrity Systems: The State of the Art", International Journal of Space Structures, Special Issue on Tensegrity Systems, 1992, 7, (2), 75-84.
[7] Hanaor, A., "Aspects of Design of Double-Layer Tensegrity Domes", International Journal of Space Structures, 1992, 7 (2), 101-113

[8] Chassagnoux, A., (2000). Shaping of Tensegrity Structures. in Structural Morphology Colloquium Proceedings: 188-193. Delft. International Association for Shell and Spatial Structures (IASS).

[9] Liapi, K. A., "Geometric Configuration and Graphical Representation of Tensegrity Spherical Networks", in ACADIA 2001: "Re-Inventing the Discourse," Proceedings, Association for Computer Aided Design in Architecture. Buffalo, New York, 2001, 258-267.

[10] Liapi, K. A., "A Novel Portable and Collapsible Tensegrity Unit for the Rapid Assembly of Tensegrity Networks", in Fifth International Conference on Space Structures, Proceedings, International Association of Space Structures, Surrey, UK, 2002, 39-46.

[11] Liapi, K.A., "A Visualization Method for the Morphological Exploration of Tensegrity Structures", in Fifth International Conference on Information Visualization (IV 200), Proceedings, IEEE Computer Society, London, UK, 2001, 232-239

[12] Liapi, K. A. and Kim, J.J, "A Parametric Approach to the Design of Vaulted Tensegrity Networks", International Journal of Architectural Computing (IJAC), pp. 88-95. 